\title{
An Ordered Weighted Averaging Operator Based on Extreme Values Reductions
}

\author{
Diego García-Zamora ${ }^{a}$, Álvaro Labella ${ }^{a}$, Rosa M. Rodríguez ${ }^{a}$ and Luis Martínez ${ }^{a}$ \\ ${ }^{a}$ Department of Computer Science,University of Jaén, Jaén, Spain, \\ \{dgzamora, alabella, rmrodrig, martin\}@ujaen.es
}

\begin{abstract}
Classical Group Decision Making (GDM) problems require the use of aggregation functions to fuse the information elicited from the experts who participate in the decision process. One of the most widely adopted aggregation functions employed in GDM are the Ordered Weighting Averaging (OWA) operators which use a linear linguistic quantifier to define the relevance of each expert. However, the use of linear quantifiers to fuse information presents some disadvantages due to the fact that they can assign the value 0 to the weights corresponding to the most extreme opinions, completely ignoring them. In this contribution, we propose a novel OWA operator which uses an Extreme Values Reduction (EVR) as linguistic quantifier to compute the relevance of each expert. The resulting operator, so-called, EVROWA operator, will always assign a non zero weight to every expert but giving greater importance to the intermediate opinions. After defining the EVR-OWA operator for a generic EVR, the EVR-OWA operators associated to two concrete families of EVRs are proposed and their main measures, i.e. mean, standard deviation, orness measure and entropy, computed. Finally an example of aggregation is developed to show the performance of the EVR-OWA operator.
\end{abstract}

Keywords: Aggregation operator, Ordered Weighted Averaging operators, Extreme Values Reduction.

\section{Introduction}

Group Decision Making (GDM) problems are those situations in which several individuals or experts pro- vide their preferences over a set of alternatives to obtain the most suitable one(s) for the problem [6]. Traditionally, a GDM process consists of two phases: (i) aggregation that aggregates the experts' preferences to obtain a collective opinion and (ii) exploitation that obtains a ranking of the alternatives to select the best one as solution of the decision problem.

Therefore, the use of aggregation operators is of vital importance in GDM. There are many aggregation operators $[1,2]$ that can be used for fusing experts' preferences in decision making, one of the most widely used is the Ordered Weighted Averaging (OWA) operator that considers the weights associated to the ordered values $[13,14]$.

In GDM problems, agreed solutions are highly appreciated [4] and in order to achieve such agreements it is necessary to include consensus reaching processes prior to the GDM solving process [9]. In such situations, it is common to find conflicts and polarized opinions among experts [3]. It has been shown that the less extreme information has a more cohesive effect and makes easier the reaching of an agreement $[10,11]$. Therefore, it seems interesting to study how to weight the extreme values in the aggregation process to achieve the consensus. Thus, our aim is to propose a novel extension of the OWA operator which uses an Extreme Values Reduction as fuzzy quantifier. The resulting aggregation operator will be characterized for assigning weights to experts' opinions depending on their degree of polarization, such that, the importance of the most extreme opinions will decrease whereas the opinions around the median value will be assigned with a higher relevance in order to facilitate the consensus reaching process. Furthermore, the abstract notion of EVR provides a generalization for other studies focused on generating weights with similar properties [12].

The structure of this contribution is as follows: Section 2 revises some basic concepts about OWA operator and 
the use of a linguistic quantifier. Section 3 introduces the new OWA operator based on Extreme Values Reductions and shows some examples to understand its performance. Section 4 presents a GDM problem that is solved by using the proposed aggregation operator. Finally, section 5 points out with some conclusions.

\section{Preliminaries}

In this section we provide a brief review about OWA operators [13] and Yager's method [14] to compute their weights from a linguistic quantifier, which will be the starting point of our proposal.

\subsection{Ordered Weighted Averaging Operators}

The Ordered Weighted Averaging (OWA) operators are a family of aggregation functions which generalizes the notion of arithmetic mean.

Definition 1 (OWA Operator [13]). Let $\omega \in[0,1]^{m}$ be a weighting vector $\left(\omega \in[0,1]^{m}, \sum_{i=1}^{m} \omega_{i}=1\right)$. The OWA Operator OWA $A_{\omega}:[0,1]^{m} \rightarrow[0,1]$ associated to $\omega$ is given by:

$$
O W A_{\omega}(\vec{x})=\sum_{k=1}^{m} \omega_{k} x_{\sigma(k)} \forall \vec{x} \in[0,1]^{m}
$$

where $\sigma$ is a permutation of the m-tuple $(1,2, \ldots, m)$ which satisfies $x_{\sigma(1)} \geq x_{\sigma(2)} \geq \ldots \geq x_{\sigma(m)}$.

Remark 1. If $\omega=(1,0, \ldots, 0) \in[0,1]^{m}$, the corresponding OWA Operator is the maximum operator, whereas when $\omega=(0,0, \ldots, 1) \in[0,1]^{m}$ the respective OWA Operator is the minimum operator. For $\omega=\left(\frac{1}{m}, \frac{1}{m}, \ldots, \frac{1}{m}\right) \in[0,1]^{m}$, the OWA Operator associated to $\omega$ is the arithmetic mean.

OWA operators have several remarkable properties such as the facts that OWA operators are idempotent non decreasing functions which are continuous, symmetric, homogeneous and shift-invariant [1].

There are several measures to study the behavior of an OWA Operator. Among the most extended measures are the arithmetic mean and the standard deviation of the weights. Other useful measures are the Orness and Entropy measures [1].

The orness measure of an OWA Operator quantifies how similar are this OWA Operator and the maximum operator. It is given by

$$
\operatorname{orness}\left(O W A_{\omega}\right)=\sum_{k=1}^{m} \omega_{k} \frac{m-k}{m-1} .
$$

When the coordinates of the weighting vector are increasing, i.e. $w_{1} \leq w_{2} \leq \ldots \leq w_{m}$, orness $\left(O W A_{\omega}\right) \in$ $\left[\frac{1}{2}, 1\right]$ whereas when these coordinates are decreasing, i.e. $w_{1} \geq w_{2} \geq \ldots \geq w_{m}$, orness $\left(O W A_{\omega}\right) \in\left[0, \frac{1}{2}\right]$.

The Entropy measure, or simply Entropy, quantifies how much information is taken into account during the aggregation process when an orness measure is set. It is given by

$$
\operatorname{Entropy}\left(O W A_{\omega}\right)=-\sum_{k=1}^{m} \omega_{k} \log \omega_{k},
$$

If no orness measure is specified, the weighting vector which maximizes the Entropy is the vector associated with the arithmetic mean operator [1].

\subsection{Using linguistic quantifiers to compute weights}

Among others proposals to compute OWA weights, Yager proposed the use of linguistic quantifiers to compute the weights for OWA Operators [14]. Let $Q$ : $[0,1] \rightarrow[0,1]$ be the linguistic quantifier for the concept of most given by

$$
Q(x)=\left\{\begin{array}{cc}
0 & 0 \leq x<\alpha \\
\frac{x-\alpha}{\beta-\alpha} & \alpha \leq x \leq \beta \\
1 & x \geq \beta
\end{array},\right.
$$

where $\alpha<\beta$ are two parameters in the interval $[0,1]$. Then, the weighting vector is computed according to the formula

$$
w_{k}=Q\left(\frac{k}{m}\right)-Q\left(\frac{k-1}{m}\right)
$$

for $k \in\{1,2, \ldots, m\}$, where $m$ is the amount of elements which we want to aggregate.

Several classic consensus processes for GDM problems $[5,7,8]$ have used this method to compute the weights of their OWA aggregations.

Let us analyze the implications of choosing the weighting vector in this way. Suppose $0<\alpha<\beta<1$. Then for any $k \leq \alpha m$ we obtain $\omega_{k}=0$. When $k \geq m \beta+1$, we also get $w_{k}=0$. If $m \alpha>k$ but $k-1 \leq m \alpha$ we obtain

$$
w_{k}=\frac{k-m \alpha}{m(\beta-\alpha)} .
$$

In the case $m \beta \leq k$ and $k-1<m \beta$ :

$$
w_{k}=1-\frac{(k-1)-m \alpha}{m(\beta-\alpha)}=\frac{m \beta-k+1}{m(\beta-\alpha)} .
$$

The remaining case $\alpha m+1<k<m \beta$ is reduced to:

$$
w_{k}=\frac{k-m \alpha}{m(\beta-\alpha)}-\frac{k-1-m \alpha}{m(\beta-\alpha)}=\frac{1}{m(\beta-\alpha)} .
$$


To summarize:

$$
w_{k}=\left\{\begin{array}{cc}
0 & 1 \leq k \leq \alpha m \\
\frac{k-m \alpha}{m(\beta-\alpha)} & m \alpha<k \leq \alpha m+1 \\
\frac{1}{m(\beta-\alpha)} & \alpha m+1<k<m \beta \\
\frac{m \beta+1-k}{m(\beta-\alpha)} & m \beta \leq k<m \beta+1 \\
0 & \beta m+1 \leq k \leq m
\end{array}\right.
$$

Note that for any $k \leq m \alpha$, all the weights $w_{k}$ are null and the same occurs when $k \geq \beta m+1$. In terms of the OWA operator associated to these weights, this fact means that the operator will ignore the first $m \alpha$ values and the last $m-(\beta m+1)$ values. In other words, the more the value of $\alpha$, the less top ranked values will be considered for the OWA aggregation. In the same way, the less the value of $\beta$, the less bottom ranked values will be considered for OWA aggregation.

In addition, the fact that there are just a few possible values for the weights is somehow against the fuzzy logic view. It should be convenient that the values of the weights change smoothly from the minimum possible value to the maximum one, instead of changing drastically from zero to $\frac{1}{m(\beta-\alpha)}$ as it occurs with the weights associated to the linguistic quantifier $Q$ for the concept of most.

Here, we will propose an alternative method to select such OWA weights which guarantees not only to take into account the more extreme values, but also allows the user to control the relevance given to these values. Our aim is to aggregate elements in a more realistic way that supports consensus reaching in polarized situations.

\section{An OWA operator based on Extreme Values Reductions}

In this section the main novelty of this contribution, namely EVR-OWA operator, is presented. This operator will be based in the notion of Extreme Value Reduction, detailed in Subsection 3.1, which are a family of automorphisms in the interval $[0,1]$ characterized for reducing the distances between the values which are close to 0 and 1. In Subsection 3.2 the EVR-OWA operator is introduced and both its arithmetic mean and its orness measure are computed. Finally, in Subsection 3.3, some examples of these EVR-OWA operators are presented.

\subsection{Extreme Values Amplifications and Extreme Values Reductions}

Those automorphisms on the interval $[0,1]$, i.e. strictly increasing bijections which satisfy the boundary condi- tions $D(0)=0$ and $D(1)=1$, characterized by increasing the distance between the values which are close to 0 and 1 will receive the name of Extreme Values Amplifications (EVAs). Formally:

Definition 2 (Extreme Values Amplification). Let D : $[0,1] \rightarrow[0,1]$ be a function satisfying:

1. D is an automorphism on the interval $[0,1]$,

2. D is a function of class $\mathscr{C}^{1}$,

3. D satisfies $D(x)=1-D(1-x) \forall x \in[0,1]$,

4. $D^{\prime}(0)>1$ and $D^{\prime}(1)>1$,

5. $D$ is concave in a neighborhood of 0 and convex in a neighborhood of 1 .

$D$ will be called then an Extreme Values Amplification (or EVA) on the interval $[0,1]$.

The main purpose of EVAs is to remap the values in the $[0,1]$ interval in a non linear way so that the new preferences show a larger distance between extreme elements and a smaller distance between elements close to $\frac{1}{2}$.

We can also state a parallel definition for those automorphisms on the interval $[0,1]$ which reduce the distance between extreme values and increases the distance between intermediate values.

Definition 3 (Extreme Values Reduction), Let $\hat{D}$ : $[0,1] \rightarrow[0,1]$ be a function satisfying:

1. $\hat{D}$ is an automorphism on the interval $[0,1]$,

2. $\hat{D}$ is a function of class $\mathscr{C}^{1}$,

3. $\hat{D}$ satisfies $\hat{D}(x)=1-\hat{D}(1-x) \forall x \in[0,1]$,

4. $\hat{D}^{\prime}(0)<1$ and $\hat{D}^{\prime}(1)<1$,

5. $\hat{D}$ is convex in a neighborhood of 0 and concave in a neighborhood of 1 ,

Then $\hat{D}$ will be called an Extreme Values Reduction (or EVR) on the interval $[0,1]$.

For both definitions, the two first properties are related with the regularity of these functions, while the third ones establish a kind of symmetry around $\frac{1}{2}$ which will be pretty useful later on.

For a better understanding of the fourth property we need a complementary result:

Theorem 1. Let $f:[a, b] \rightarrow \mathbb{R}$ be a $\mathscr{C}^{1}$ function defined on the interval $[a, b] \subset \mathbb{R}$. Then the following statements are equivalent: 
1. $f$ is an increasing function satisfying $\mid f(y)-$ $f(x)|\geq| y-x \mid \forall x, y \in[a, b]$,

2. $f^{\prime}(z) \geq 1 \forall z \in[a, b]$.

Proof. $1 \rightarrow 2)$ Suppose first that $|f(y)-f(x)| \geq \mid y-$ $x \mid \forall x, y \in[a, b]$ and pick $z \in[a, b[$. Let us choose $h>0$ such that $z+h<b$. In that case the auxiliary function $g:] 0, h\left[\rightarrow \mathbb{R}\right.$ defined by $\left.g(t)=\frac{f(z+t)-f(z)}{t} \forall t \in\right] 0, h[$ is a continuous function such that $g(t) \geq 1 \forall t \in] 0, h[$ and therefore $f^{\prime}(z)=\lim _{t \rightarrow 0} g(t) \geq 1$. On the other hand, when $z=b$, we can consider $h>0$ such that $b-a>$ $h$ and use the analogue reasoning for the function $g$ : ] $0, h\left[\rightarrow \mathbb{R}\right.$ defined by $\left.g(t)=\frac{f(b)-f(b-t)}{t} \forall t \in\right] 0, h[$.

$2 \rightarrow 1)$ Since $f^{\prime}(z) \geq 1 \forall z \in[a, b], f$ is increasing. To show the inequality pick $x, y \in[a, b]$ such that $x<y$. We can use the Mean Value Theorem to obtain $\xi \in] x, y[$ such that $f(y)-f(x)=f^{\prime}(\xi)(y-x)$ and therefore it must be $|f(y)-f(x)| \geq|y-x|$.

In other words, $f^{\prime} \geq 1$ implies distance amplifications while $f^{\prime} \leq 1$ implies a distance reduction after applying the function $f$.

The following result will be the key for constructing our new OWA operator by using the fifth property.

Proposition 1. Let $f:[a, b] \rightarrow \mathbb{R}$ be a differentiable increasing function. The following statements are equivalent:

1. $f$ is a convex function,

2. For each $x<y, x, y \in] a, b]$ the inequality

$$
|f(x)-f(x-t)| \leq|f(y)-f(y-t)|
$$

holds for any $t \in[0, h]$, where $h=$ $\min \{x-a, y-x\}$.

Proof. 1) $\rightarrow 2$ ). If $f$ is convex, $f^{\prime}$ is an increasing function in $[a, b]$. On the other hand, the Mean Value Theorem gives us $\left.\xi_{1} \in\right] x-t, x\left[\right.$ and $\left.\xi_{2} \in\right] y-t, y[$ such that

$$
\begin{array}{r}
|f(x)-f(x-t)|=f(x)-f(x-t)=f^{\prime}\left(\xi_{1}\right) t \leq \\
f^{\prime}\left(\xi_{2}\right) t=f(y)-f(y-t)=|f(y)-f(y-t)|,
\end{array}
$$

where we have used that $f^{\prime}$ is increasing and therefore $f^{\prime}\left(\xi_{1}\right) \leq f^{\prime}\left(\xi_{2}\right)$.

$2) \rightarrow 1)$. Fix $x<y, x, y \in] a, b]$ and define $g:[0, h] \rightarrow \mathbb{R}$ by

$$
\left.\left.g(t)=\frac{f(y)-f(y-t)-(f(x)-f(x-t))}{t} \forall t \in\right] 0, h\right] .
$$

Since $f$ is increasing $g \geq 0$. In addition

$$
\lim _{t \rightarrow 0} g(t)=f^{\prime}(y)-f^{\prime}(x)
$$

and the continuity of $g$ leads to $f^{\prime}(y) \geq f^{\prime}(x)$, which is the convexity of $f$.

Remark 2. The inequality in the previous result cannot be replaced for a strict inequality, because though $f^{\prime}$ is increasing, we cannot assure it is strictly increasing.

We can obtain a similar result for concave functions:

Proposition 2. Let $f:[a, b] \rightarrow \mathbb{R}$ be a differentiable increasing function. The following statements are then equivalent:

1. $f$ is a concave function,

2. For each $x<y, x, y \in] a, b]$ the inequality

$$
|f(x)-f(x-t)| \geq|f(y)-f(y-t)|
$$

holds for any $t \in[0, h]$, where $h=$ $\min \{x-a, y-x\}$.

\subsection{A novel EVR-OWA Operator}

Here, we will use the fifth property of EVRs to construct an OWA Operator which has similar measures to the arithmetic mean but giving more importance to the intermediate values and less importance to the more extreme values to smooth out the importance of polarized opinions in GDM, but taking them into account instead of ignoring them.

Let us start by clarifying this property. Consider an EVR $\hat{D}:[0,1] \rightarrow[0,1]$ which is twice differentiable and convex in $[0,0.5]$ and twice differentiable and concave in $[0.5,1]$. Suppose we have a partition of the interval $[0,1]$. For instance, we can take $m \in \mathbb{N}$ and define

$$
x_{k}=\frac{k}{m}, \forall k \in\{1,2, \ldots, m\}
$$

Since $\hat{D}$ is convex in $[0,0.5]$, this fifth property assures that, for any $k_{1} \leq k_{2}$ such that $x_{k_{1}}, x_{k_{2}} \in\left[\frac{1}{m}, \frac{1}{2}\right]$ we obtain

$$
\left|\hat{D}\left(x_{k_{1}}\right)-\hat{D}\left(x_{k_{1}}-\frac{1}{m}\right)\right| \leq\left|\hat{D}\left(x_{k_{2}}\right)-\hat{D}\left(x_{k_{2}}-\frac{1}{m}\right)\right| .
$$

In other words, the more closer $x_{k}$ is to 0 , the smaller the difference $\left|\hat{D}\left(x_{k}\right)-\hat{D}\left(x_{k}-\frac{1}{m}\right)\right|$. A similar reasoning leads us to the concave counterpart of this: the more closer $x_{k}$ is to 1 , the smaller the difference $\left|\hat{D}\left(x_{k}\right)-\hat{D}\left(x_{k}-\frac{1}{m}\right)\right|$.

This fact is utilized to define the weights of an OWA operator by using the general scheme introduced by Yager [14]. 
Definition 4 (EVR-OWA Operator). Let $\hat{D}$ be an Extreme Values Reduction and consider $m \in \mathbb{N}$. We define

$$
w_{k}=\hat{D}\left(\frac{k}{m}\right)-\hat{D}\left(\frac{k-1}{m}\right) \forall k \in\{1,2, \ldots, m\} .
$$

The family $W=\left\{w_{1}, w_{2}, \ldots, w_{m}\right\}$ will receive the name of order $m$ weights associated with the EVR $\hat{D}$, and the OWA- operator given by $\Phi_{W}:[0,1]^{m} \rightarrow[0,1]$ defined by

$$
\begin{array}{r}
\Phi_{W}\left(x_{1}, x_{2}, \ldots, x_{m}\right)=\sum_{k=1}^{m} w_{k} x_{\sigma(k)}, \\
\forall\left(x_{1}, x_{2}, \ldots, x_{m}\right) \in[0,1]^{m},
\end{array}
$$

where $\sigma$ is a permutation of the m-tuple $(1,2, \ldots, m)$ which satisfies $x_{\sigma(1)} \geq x_{\sigma(2)} \geq \ldots \geq x_{\sigma(m)}$, will be called the EVR-OWA operator associated with $\hat{D}$ to aggregate $m$ elements.

Remark 3. Since $\hat{D}$ is an automorphism of $[0,1]$, we can consider it as a fuzzy quantifier and so we can repeat the scheme introduced by Yager.

We highlight the philosophy behind this operator. As any OWA operator, $\Phi_{W}$ starts by ordering the values $x_{1}, x_{2}, \ldots, x_{m}$ from the largest one, to the smallest one. When we use these weights, those values of $x$ which are closer to extremes, i.e. the largest ones, and the smallest ones, are matched with the smallest weights while the intermediate values of $x$ will be matched with the highest $w_{k}$ 's. Hence, this operator aggregates elements by assigning more relevance to the intermediate values of the elements which aggregates, and giving less importance to the more extreme elements, but taking them into account since $\hat{D}$ is strictly increasing and therefore those weights can not be null.

Let us analyze some properties of these weights. First note that since $\hat{D}$ is strictly increasing, all of these weights are greater than zero. In addition,

$$
\begin{array}{r}
\sum_{k=1}^{m} w_{k}=\sum_{k=1}^{m} \hat{D}\left(\frac{k}{m}\right)-\hat{D}\left(\frac{k-1}{m}\right)= \\
=\hat{D}(1)-\hat{D}(0)=1,
\end{array}
$$

and they are properly defined.

By using the third condition of the EVR definition, i.e. $\hat{D}(x)=1-\hat{D}(1-x) \forall x \in[0,1]$, we get:

$$
\begin{array}{r}
\hat{D}\left(\frac{k}{m}\right)=1-\hat{D}\left(1-\frac{k}{m}\right)=1-\hat{D}\left(\frac{m-k}{m}\right), \\
\hat{D}\left(\frac{k-1}{m}\right)=1-\hat{D}\left(1-\frac{k-1}{m}\right)=1-\hat{D}\left(\frac{m-k+1}{m}\right),
\end{array}
$$

therefore

$$
\begin{array}{r}
w_{k}=\hat{D}\left(\frac{k}{m}\right)-\hat{D}\left(\frac{k-1}{m}\right)= \\
=\hat{D}\left(\frac{m-k+1}{m}\right)-\hat{D}\left(\frac{m-k}{m}\right)=w_{m-k+1} .
\end{array}
$$

This symmetry and the fifth property of EVRs, used as we have explained before, give us an idea of the distribution of these weights. On the one hand, the smallest values of $w_{k}$ are always located at $k=1$ and $k=m$, i.e.

$$
w_{\text {min }}=w_{1}=D\left(\frac{1}{m}\right)=1-D\left(1-\frac{1}{m}\right)=w_{m} .
$$

We know that these weights are matched by pairs. So there is a minimum value at $w_{1}$ and the values of the weights will strictly increase until a certain maximum value $w_{\max }$ and then, because of the symmetry $w_{k}=w_{m-k+1}$, the values of the weights will start to decrease towards the value $w_{m}=w_{1}$. The maximum value for $w_{k}$ depends on the parity of $m$. When $m$ is even, the maximum value will be at $k=\frac{m}{2}$ due to $w_{\frac{m}{2}}=w_{m-\frac{m}{2}+1}=w_{\frac{m+2}{2}}$. When $m$ is odd, the maximum value is at $k=\frac{m+1}{2}$ :

$$
w_{\max }=\left\{\begin{array}{cc}
w_{\frac{m}{2}} & \text { if } m \text { is even } \\
w_{\frac{m+1}{2}} & \text { if } m \text { is odd }
\end{array},\right.
$$

Let us remark here that $w_{\max }$ is a kind of median value for the weights.

Note that the arithmetic mean of these weights is

$$
w_{0}=\frac{1}{m} \sum_{k=1}^{m} w_{k}=\frac{1}{m}
$$

To compute the orness measure we have to distinguish two cases. If $m$ is even:

$$
\begin{array}{r}
\operatorname{orness}\left(\Phi_{\hat{D}}\right)=\sum_{k=1}^{m} w_{k} \frac{m-k}{m-1}= \\
\sum_{k=1}^{m / 2} w_{k} \frac{m-k}{m-1}+\sum_{k=m / 2+1}^{m} w_{k} \frac{m-k}{m-1}= \\
\sum_{k=1}^{m / 2} w_{k} \frac{m-k}{m-1}+\sum_{k=m / 2+1}^{m} w_{m-k+1} \frac{m-k}{m-1}= \\
\sum_{k=1}^{m / 2} w_{k} \frac{m-k}{m-1}+\sum_{k=m / 2}^{1} w_{k} \frac{k-1}{m-1}= \\
\sum_{k=1}^{m / 2} w_{k} \frac{m-k}{m-1}+\sum_{k=1}^{m / 2} w_{k} \frac{k-1}{m-1}= \\
\sum_{k=1}^{m / 2} w_{k} \frac{m-k}{m-1} w_{k} \frac{k-1}{m-1}= \\
\sum_{k=1}^{m / 2} w_{k}=D(1 / 2)=\frac{1}{2} .
\end{array}
$$


If $m$ is odd

$$
\begin{aligned}
\operatorname{orness}\left(\Phi_{\hat{D}}\right)=\sum_{k=1}^{m} w_{k} \frac{m-k}{m-1} & = \\
\sum_{k=1}^{(m-1) / 2} w_{k}+w_{(m+1) / 2} \frac{2 m-(m-1)}{2(m-1)} & = \\
=D\left(\frac{m-1}{2 m}\right)+\frac{1}{2}\left(D\left(\frac{m+1}{2 m}\right)-D\left(\frac{(m-1) / 2-1}{m}\right)\right) & = \\
\frac{1}{2}\left(D\left(\frac{m+1}{2 m}\right)+D\left(\frac{m-1}{2 m}\right)\right) & =\frac{1}{2}
\end{aligned}
$$

So orness $\left(\Phi_{\hat{D}}\right)=0.5$ for any $\operatorname{EVR} \hat{D}$ and any $m \in \mathbb{N}$.

\subsection{Examples of EVR-OWA operators}

In this subsection two families of EVRs are introduced, namely the $\sin$ based EVR $s_{\alpha}$ and the polynomial based $m_{\alpha}$. For the EVR-OWA operator associated to these families, the entropy measure, the standard deviation (SD) and both the minimum and maximum values for the weights are computed.

\section{The EVR-OWA associated to $s_{\alpha}$}

Let $\alpha \in] 0, \frac{1}{2 \pi}\left[\right.$ and consider the $\mathscr{C}^{\infty}$ EVR $s_{\alpha}:[0,1] \rightarrow$ $[0,1]$ given by

$$
\hat{s}_{\alpha}(x)=x+\alpha \cdot \sin (2 \pi x-\pi) \forall x \in[0,1] .
$$

For $m=100$, table 1 shows the calculations of the most standard measures for the weights obtained for different values of $\alpha$ (keep in mind that for $\alpha=0$ we get the weights associated to the arithmetic mean operator). In figure 1 we show the comparison between values of the weights obtained for several values of $\alpha$.

Table 1: Measures for $\hat{s}_{\alpha}, m=100$

\begin{tabular}{|c|c|c|c|c|c|c|}
\hline EVA & Orness & Entropy & Mean & SD & Min & Max \\
\hline$\hat{s}_{0}$ & 0.5 & 4.6051 & 0.01 & 0 & 0.01 & 0.01 \\
$\hat{s}_{0.05}$ & 0.5 & 4.5801 & 0.01 & 0.0022 & 0.0068 & 0.0131 \\
$\hat{s}_{0.08}$ & 0.5 & 4.5398 & 0.01 & 0.0035 & 0.0049 & 0.0150 \\
$\hat{s}_{0.09}$ & 0.5 & 4.5216 & 0.01 & 0.0039 & 0.0043 & 0.0156 \\
$\hat{s}_{0.15}$ & 0.5 & 4.3443 & 0.01 & 0.0066 & 0.0005 & 0.0194 \\
\hline
\end{tabular}

\section{The EVR-OWA associated to $m_{\alpha}$}

Let $\alpha>1$ and consider the EVR $\hat{m}_{\alpha}:[0,1] \rightarrow[0,1]$ given by

$$
\hat{m}_{\alpha}(x)=\left\{\begin{array}{ll}
\frac{1}{2}-\frac{1}{2}(1-2 x)^{\frac{1}{\alpha}} & 0 \leq x<\frac{1}{2} \\
\frac{1}{2}+\frac{1}{2}(2 x-1)^{\frac{1}{\alpha}} & \frac{1}{2} \leq x \leq 1
\end{array} .\right.
$$

Remark 4. Note that $\hat{m}_{\alpha}$ is not an EVR strictly speaking since it is not differentiable in $x=\frac{1}{2}$. However, $\lim _{x \rightarrow \frac{1}{2}-} f^{\prime}(x)=\lim _{x \rightarrow \frac{1}{2}+} f^{\prime}(x)=+\infty$ and therefore it will behave as an EVR.

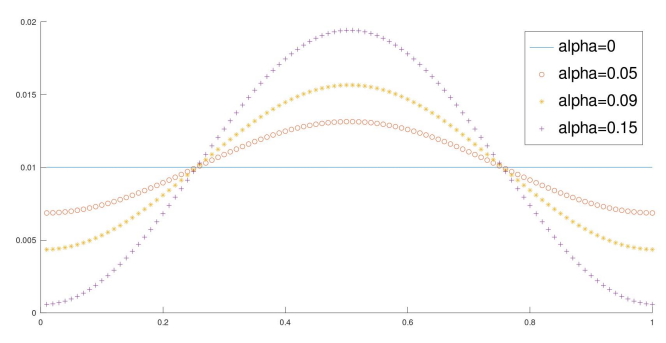

Figure 1: Comparison of $w_{k}$ for different $\hat{s}_{\alpha}, m=100$.

For $m=100$ we show the results of the calculations for most standard measures for the weights in table 2 (in this case, the weights associated to the arithmetic mean operator are given by $\alpha=1$ ). In Figure 2 we show the comparison between values of the weights obtained for the different values of $\alpha$.

Table 2: Measures for $\hat{m}_{\alpha}, m=100$

\begin{tabular}{|c|c|c|c|c|c|c|}
\hline EVA & Orness & Entropy & Mean & SD & Min & Max \\
\hline$\hat{m}_{1}$ & 0.5 & 4.605 & 0.01 & 0 & 0.01 & 0.01 \\
$\hat{m}_{1.35}$ & 0.5 & 4.5581 & 0.01 & 0.0034 & 0.0074 & 0.0275 \\
$\hat{m}_{2}$ & 0.5 & 4.3421 & 0.01 & 0.0098 & 0.0050 & 0.0707 \\
$\hat{m}_{3.39}$ & 0.5 & 3.8057 & 0.01 & 0.0219 & 0.0029 & 0.1576 \\
$\hat{m}_{5}$ & 0.5 & 3.3090 & 0.01 & 0.0317 & 0.0020 & 0.2286 \\
$\hat{m}_{10}$ & 0.5 & 2.4379 & 0.01 & 0.0470 & 0.0010 & 0.3381 \\
\hline
\end{tabular}

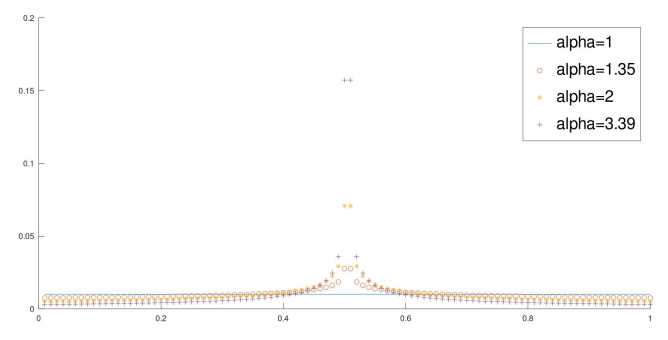

Figure 2: Comparison of $w_{k}$ for different $\hat{m}_{\alpha}, m=100$.

\section{An illustrative example}

Suppose a group consisting on 5 experts who elicit their preferences on how much they prefer a certain alternative $x_{1}$ to the alternative $x_{2}$. These preferences will be denoted as $p_{k}:=p_{1,2}^{k} \in[0,1], k=1,2, \ldots, 5$ are 
given by:

$$
\begin{aligned}
& p_{1}=0.6 \\
& p_{2}=0.9 \\
& p_{3}=0.8 \\
& p_{4}=0.1 \\
& p_{5}=0.3
\end{aligned}
$$

For $m=5$ and the EVR $\hat{s}_{0.15}$ the obtained list of weights is:

$$
\begin{aligned}
& w_{1}=0.0573 \\
& w_{2}=0.2544 \\
& w_{3}=0.3763 \\
& w_{4}=0.2544 \\
& w_{5}=0.0573
\end{aligned}
$$

and the collective preference by using these weights is given by:

$$
\begin{array}{r}
p_{1,2}=<\left(p_{1}, p_{2}, p_{3}, p_{4}, p_{5}\right) \searrow,\left(w_{1}, w_{2}, w_{3}, w_{4}, w_{5}\right)>= \\
<\left(p_{2}, p_{3}, p_{1}, p_{5}, p_{4}\right) \searrow,\left(w_{1}, w_{2}, w_{3}, w_{4}, w_{5}\right)>= \\
=0.0573 \cdot 0.9+0.2544 \cdot 0.8+0.3763 \cdot 0.6 \\
+0.2544 \cdot 0.3+0.0573 \cdot 0.1=0.5629
\end{array}
$$

Table 3 compiles the standard measures for both the arithmetic mean $\left(\hat{s}_{0}\right)$ and the EVR-OWA associated to $\hat{s}_{0.15}$.

Note that the arithmetic mean operator assigns a weight of 0.2 to every expert when $m=5$ while the weights corresponding to the EVR-OWA take values in the interval $[0.0573,0.3763]$. The highest weight is associated to the median value of the preferences whereas the most extreme values receives the minimum weight.

These changes in the values of the weights are translated into a smaller value for the entropy and a slight increment for the value of the standard deviation.

Figure 3 provides a graphical comparison between the weights obtained for arithmetic mean operator, i.e. $\hat{s}_{0}=0$, and the weights corresponding to the EVROWA associated with $\hat{s}_{0.15}$.

\section{Conclusions}

The use of linguistic quantifiers to define OWA operators throughout Yager's method presents some limitations depending on the values of the parameters $\alpha$ and
Table 3: Measures for $\hat{s}_{0}$ and $\hat{s}_{0.15}, m=5$

\begin{tabular}{|c|c|c|c|c|c|c|}
\hline EVA & Orness & Entropy & Mean & SD & Min & Max \\
\hline$\hat{s}_{0}$ & 0.5 & 1.60 & 0.2 & 0 & 0.2 & 0.2 \\
$\hat{s}_{0.15}$ & 0.5 & 1.3921 & 0.2 & 0.1246 & 0.0573 & 0.3763 \\
\hline
\end{tabular}

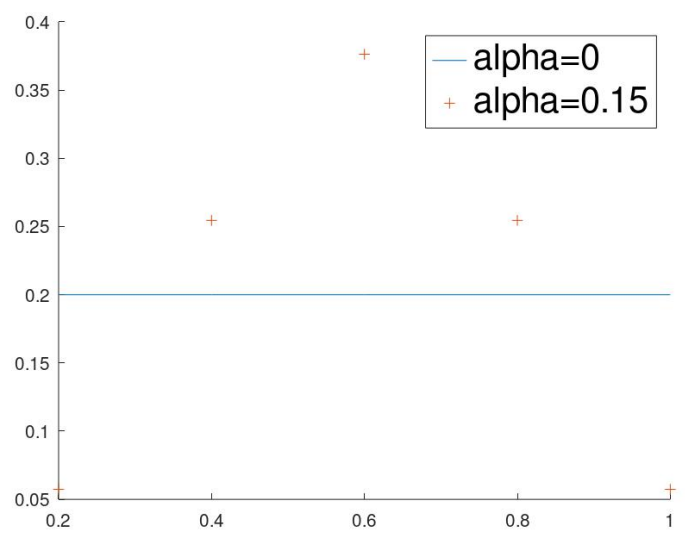

Figure 3: Comparison of 5 experts' $w_{k}$ 's for $\hat{s}_{\alpha}$ with $\alpha=0$ and $\alpha=0.15$.

$\beta$. One of these limitations is the fact that, with just two possible exceptions, the value $w_{k}$ is either null or the constant $\frac{1}{m(\beta-\alpha)}$. Therefore, the progressive behavior of the weighting vector calculated as we propose in this contribution is more related to the fuzzy environment we are dealing with: whereas the classical quantifier $Q$ completely ignores the most extreme elements, the EVR quantifier we propose will provide a progressive reduction of the importance we are giving to the more extreme values, which is closer to the fuzzy philosophy.

By using the OWA operator associated to an EVR we can overcome these limitations obtaining an OWA operator which takes into account the more extreme values, but gives more importance to the intermediate ones. By adjusting the parameters of the EVR, we can control the balance between the relevance given to the extreme values and the intermediate ones.

To summarize, we obtain weighting vectors for OWA operators which assign small relevance to the most extreme elements and give more importance to the intermediate elements. The first values of the weights together with the last ones will be the more extreme, and therefore will receive the lower values of the weights. These weights will progressively increase until they reach the maximum value at approximately the median element to be aggregated. Then, the values of the weights will progressively decrease towards the mini- 
mum value at $w_{m}=w_{1}$

Further studies should be related with the behavior of these weights in large scale GDM problems and consensus reaching processes in which preferences tends to be polarized.

\section{Acknowledgement}

This work is partially supported by the Spanish Ministry of Economy and Competitiveness through the Spanish National Project PGC2018-099402-B-I00, and the Postdoctoral fellow Ramón y Cajal (RYC2017-21978), and by the Spanish Ministry of Science, Innovation and Universities through a FPU (Formación de Profesorado Universitario) grant.

\section{References}

[1] G. Beliakov, H. Bustince, T. Calvo, A practical guide to averaging functions, Springer, 2016.

[2] G. Beliakov, A. Pradera, T. Calvo, Aggregation Functions: A Guide for Practitioners, Springer, 2007.

[3] Q. Dong, X. Zhou, L. Martínez, A hybrid group decision making framework for achieving agreed solutions based on stable opinions, Information Sciences 490 (2019) 227-243.

[4] P. Eklund, A. Rusinowska, H. De Swart, A consensus model of political decision-making, Annals of Operations Research 158 (1) (2008) pp. 5-20.

[5] E. Herrera-Viedma, F. Herrera, F. Chiclana, A consensus model for multiperson decision making with different preference structures, Systems, Man and Cybernetics, Part A: Systems and Humans, IEEE Transactions on 32 (2002) $394-402$.

[6] J. Kacprzyk, Group decision making with a fuzzy linguistic majority, Fuzzy Sets Syst 18 (2) (1986) $105-118$.

[7] Á. Labella, Y. Liu, R. M. Rodríguez, L. Martínez, Analyzing the performance of classical consensus models in large scale group decision making: A comparative study, Applied Soft Computing 67 (2018) 677-690.

[8] I. Palomares, F. J. Estrella, L. Martínez, F. Herrera, Consensus under a fuzzy context: Taxonomy, analysis framework afryca and experimental case of study, Information Fusion 20 (2014) 252-271.
[9] R. M. Rodríguez, Á. Labella, G. D. Tré, L. Martínez, A large scale consensus reaching process managing group hesitation, KnowledgeBased Systems 159 (2018) 86-97.

[10] A. Sîrbu, V. Loreto, V. Servedio, Opinion dynamics with disagreement and modulated information, Journal of Statistical Physics 151 (2013) 218-237.

[11] A. Sîrbu, V. Loreto, V. Servedio, F. Tria, Cohesion, consensus and extreme inormation in opinion dynamics, Advances in Complex Systems 16 (06) (2013) 1350035.

[12] Z. Xu, An overview of methods for determining owa weights, International Journal of Intelligent Systems 20 (8) (2005) 843-865.

[13] R. Yager, Families of OWA operators, Fuzzy Sets and Systems 59 (2) (1993) 125 - 148

[14] R. R. Yager, Quantifier guided aggregation using OWA operators, International Journal of Intelligent Systems 11 (1) (1996) 49-73. 